Proceedings of the 43rd "Jaszowiec", International School and Conference on the Physics of Semiconductors, Wisła 2014

\title{
Thermoelectric Transport through the Double Quantum Dot in the Sequential Tunneling Regime
}

\author{
B. SzUKIEWICZ AND K.I. WYSOKIŃSKI* \\ Institute of Physics, M. Curie-Skłodowska University, I. Radziszewskiego 10, PL 20-031 Lublin, Poland \\ The charge and spin thermoelectric transport in the system composed of a central molecule in contact with two \\ normal electrodes in an external magnetic field of the Zeeman type have been studied. Such system can support \\ pure spin current for applications in spintronics. By appropriate gate tuning of each of the dots it is possible to \\ electrically control the direction of the spin current or tune the device operating as a thermoelectric generator to \\ optimal performance.It has been shown that the device is poor energy converter in the parameter region where its \\ thermoelectric figure of merit attains very large values.
}

DOI: 10.12693/APhysPolA.126.1159

PACS: 73.23.-b, 73.63.Kv, 73.23.Hk

\section{Introduction}

Recent experimental advances in nanotechnology has sparked a lot of work on quantum dots and molecules. As a result the control of the single photon, electron or spin has been achieved. One of the perspective directions of nanotechnology is building devices which can operate as efficient energy harvesters or refrigerators. Traditionally it is the usage of the Seebeck effect, which allows energy generation from waste heat. The Seebeck effect is quantified by the Seebeck coefficient $S$ which is a voltage $V$ generated in the system by the temperature difference $\delta T=T_{\mathrm{h}}-T_{\mathrm{c}}$ between hot and cold sides of it under open circuit condition: $S=-(V / \delta T)_{I=0}$. The useful materials are characterized not only by large values of the Seebeck coefficient $S$ but also by large thermoelectric figure of merit ZT. ZT is defined as ZT $=G S^{2} T / \kappa$, where $G$ is the conductance, $\kappa$ - thermal conductance and $T$ - temperature and requires good thermoelectric performance, but small thermal conductivity [1].

The largest $\mathrm{ZT}$ is obtained for the devices operating in a reversible way when their output power vanishes. Thus the mere increase of ZT in nanodevices is useless unless their output power is finite. The goal is thus to optimise the operation of nanoengines and to find the conditions for obtaining the maximum power with the maximum possible efficiency. The other important direction of study is the control of the spin and its flow i.e. the spin current. The spin counterpart of the Seebeck effect has been found [2] very useful to experimentally obtain and control the flow of pure spin current. The efficiency of the heat to spin voltage conversion [3] depends on the spin Seebeck coefficient $S_{\mathrm{s}}$.

Here we shall use the model of the central system which may describe single level quantum dot with quadruple degeneracy (like the carbon nanotube quantum dot), a two level quantum dot or two single level quantum dots

${ }^{*}$ corresponding author; e-mail: karol@tytan.umcs.lublin.pl in parallel. In the last case the central system is called quantum dot molecule and intensively studied in the limit of the Coulomb blockade $[4,5]$ with the goal to find configurations with enhanced Seebeck coefficient $S_{\mathrm{c}}$ or thermoelectric figure of merit $\mathrm{Z}_{\mathrm{c}} \mathrm{T}[6,7]$ or their spin counterparts i.e. spin Seebeck coefficient $S_{\mathrm{s}}$ and spin thermoelectric figure of merit $Z_{\mathrm{s}} \mathrm{T}[8,9]$.

In this paper we study thermally induced charge, spin and heat flow in a sequential tunneling limit. In this limit we use master equation approach to calculate currents. The method is not able to describe the Kondo effect but it treats Coulomb interactions between electrons exactly and the coupling between the central region and external leads to the first order. Beside the charge and spin currents we shall also calculate the performance of the system as an energy harvesting device. Such a device takes heat from the hot lead performs work and releases rest heat to cold reservoir. The efficiency of the device at the maximum power [10] is a typical question one asks studying real engines. The efficiency of the engine is defined as the ratio of power $P$ to the heat flux from the hot reservoir $\eta=P / \dot{Q}_{\mathrm{h}}$. For the reversibly operating heat engine $\eta$ is limited by the Carnot value $\eta_{\mathrm{C}}=\delta T / T_{\mathrm{h}}$. The efficiency of endoreversible heat engine at maximum power is given by the Curzon-Ahlborn [10] limit $\eta_{\mathrm{CA}}=1-\sqrt{1-\eta_{\mathrm{C}}}$.

\section{The model and approach}

The interacting quantum dots are described by the Hamiltonian

$$
H=H_{\mathrm{el}}+H_{\mathrm{DQD}}+H_{\mathrm{T}},
$$

where

$$
H_{\mathrm{el}}=\sum_{\boldsymbol{k}, \nu=\mathrm{L}, \mathrm{R}} \sum_{\sigma=\uparrow, \downarrow}\left(\varepsilon_{\nu \boldsymbol{k}}-\mu_{\nu}\right) c^{\dagger}{ }_{\nu \boldsymbol{k} \sigma} c_{\nu \boldsymbol{k} \sigma}
$$

is the energy operator for external electrodes. The central system is described by the Hamiltonian

$$
\begin{aligned}
& H_{\mathrm{DQD}}=\sum_{i=1,2} \sum_{\sigma=\uparrow, \downarrow} \varepsilon_{i \sigma} d_{i \sigma}^{\dagger}{ }_{i \sigma} d_{i \sigma} \\
& \quad+U_{1} n_{1 \uparrow} n_{1 \downarrow}+U_{2} n_{2 \uparrow} n_{2 \downarrow}+\sum_{\sigma, \sigma \prime} U_{12} n_{1 \sigma} n_{2 \sigma},
\end{aligned}
$$


while the coupling term between external leads and the central part reads

$$
H_{\mathrm{T}}=\sum_{i=1,2} \sum_{\boldsymbol{k}, \nu=\mathrm{L}, \mathrm{R}} \sum_{\sigma=\uparrow, \downarrow}\left(\gamma_{i \nu \boldsymbol{k}} c^{\dagger}{ }_{\nu \boldsymbol{k} \sigma} d_{i \sigma}+\text { H.c. }\right) .
$$

The operators $d_{i \sigma}^{\dagger}\left(d_{i \sigma}\right)$ create (annihilate) electron of spin $\sigma$ on the dot $i=1,2$, while $c_{\nu \boldsymbol{k} \sigma}^{\dagger}\left(c_{\nu \boldsymbol{k} \sigma}\right)$ create (annihilate) spin $\sigma$ electrons in the state $\boldsymbol{k}$ of electrode $\nu$. $n_{\sigma}=d_{\sigma}^{\dagger} d_{\sigma}$ is the number operator. The $\sigma$ dependence of the on-dot energy $\varepsilon_{d \sigma}$ is due to the external magnetic field: $\varepsilon_{d \sigma}=\varepsilon_{d}+g_{\sigma} \mu_{B} B, g_{\uparrow}=1, g_{\downarrow}=-1$. The effective couplings between the $i$-th dot and $\nu$-th electrode

$$
\Gamma_{i}^{\nu}=\sum_{\boldsymbol{k}} \gamma_{i \nu \boldsymbol{k}} \delta\left(\varepsilon-\varepsilon_{\nu \boldsymbol{k}}\right)
$$

is energy independent in the wide band limit. In the following we shall use units with electron charge $e$, Boltzmann constant $k_{\mathrm{B}}$ and Bohr magneton $\mu_{\mathrm{B}}$ all equal to 1 and take the coupling to the left lead as an energy unit $\Gamma_{\mathrm{L}}=\Gamma=1$. In this work we shall assume spin independent couplings and non-magnetic electrodes.

In the master equation approach one first calculates the probabilities $P_{\alpha}$ of the central region to be in a given quantum state $|\alpha\rangle$. For our system it is convenient to write $|\alpha\rangle=|n, m\rangle$, where $n, m$ denote the occupation of the lower, upper (level in two level quantum dot or lower, upper quantum dot in a quantum dot molecule). The master equation for the probabilities is written as

$$
\frac{\mathrm{d} P_{\alpha}}{\mathrm{d} t}=-\sum_{\alpha \neq \beta} W_{\beta \alpha} P_{\alpha}+\sum_{\beta \neq \alpha} W_{\alpha} P_{\beta},
$$

where the symbols $W_{\alpha \beta}\left(W_{\beta \alpha}\right)$ denote the total transition rates from initial state $\beta(\alpha)$ to final state $\alpha(\beta)$. They are sums of the (in) and (out) rates for all leads. For calculating the currents we need the probabilities in the stationary limit $\mathrm{d} P_{\alpha} / \mathrm{d} t=0$. As each level in two dots may be empty, singly (spin up or spin down) or doubly occupied, we end up with $16 \times 16$ problem. After finding all probabilities we calculate spin resolved charge currents $I_{\sigma}^{\nu}$ flowing from the electrode $\nu=\mathrm{L}, \mathrm{R}$ towards central region. Similarly we calculate heat flow for each electrode $I_{Q}^{\nu}$ towards the central molecule. The charge and energy of the central region are conserved. This means that $I_{\mathrm{c}}^{\mathrm{L}}=-I_{\mathrm{c}}^{\mathrm{R}}=I_{\mathrm{c}}$ and $\left(\mu_{\mathrm{L}}-\mu_{\mathrm{R}}\right) I_{\mathrm{c}}+I_{Q}^{\mathrm{R}}+I_{Q}^{\mathrm{L}}=0$. The first term here, i.e. $\left(\mu_{\mathrm{L}}-\mu_{\mathrm{R}}\right) I_{\mathrm{c}}=P$ is the power of the engine. We assumed that that the $\mathrm{R}(\mathrm{L})$ electrode is hot (cold) with temperature $T_{\mathrm{R}(\mathrm{L})}=T+(-) \delta T / 2$ and the voltages $\mu_{\mathrm{R}(\mathrm{L})}=\mu-(+) V / 2$.

The knowledge of the spin resolved currents in the external leads allows us to calculate total spin current flowing in the system $I_{\mathrm{s}}=I_{\uparrow}-I_{\downarrow}$. If spin relaxation time in the electrodes is long enough, the spin dependent chemical potentials $\mu_{\alpha \sigma}$ have to be considered. Under this condition one may define [6] the spin voltage (or spin bias) $e \Delta V_{\mathrm{s}}=\left(\Delta \mu_{\uparrow}-\Delta \mu_{\downarrow}\right) / 2$ and the (charge) voltage $e \Delta V=\left(\Delta \mu_{\uparrow}+\Delta \mu_{\downarrow}\right) / 2$. Then both charge and spin currents depend on voltage $\Delta V$ and spin voltage $\Delta V_{\mathrm{s}}$ and the spin dependent transport coefficients $\mathcal{L}_{i j}$ may be easily calculated. Here we limit our discussion to the currents flowing in the system and delegate the analysis of $\mathcal{L}_{i j}$, Seebeck coefficients, etc. to the forthcoming paper [11].

\section{Charge and spin currents}

Our main results are shown in Figs. 1-3 below. The upper part of Fig. 1 shows $I_{\mathrm{c}}$ and $I_{\mathrm{s}}$ of the single level quantum dot in an external magnetic field $B$. Already this simple device allows for pure spin current [3]. For the non-interacting dot the spin current (curves with symbols) is maximal at the particle-hole symmetry point $\varepsilon_{\mathrm{d}}=0$ when charge current exactly vanishes (for all $B$ values). The spin up electrons flow from left to right, while the same flux of spin down electrons goes opposite direction. As a result the charge current vanishes, while spin current is maximal. In a two level quantum dot with the distance between levels $\varepsilon_{d 1}-\varepsilon_{d 2}=1 \Gamma$ (lower part in Fig. 1) there exist three points (marked by arrows in the

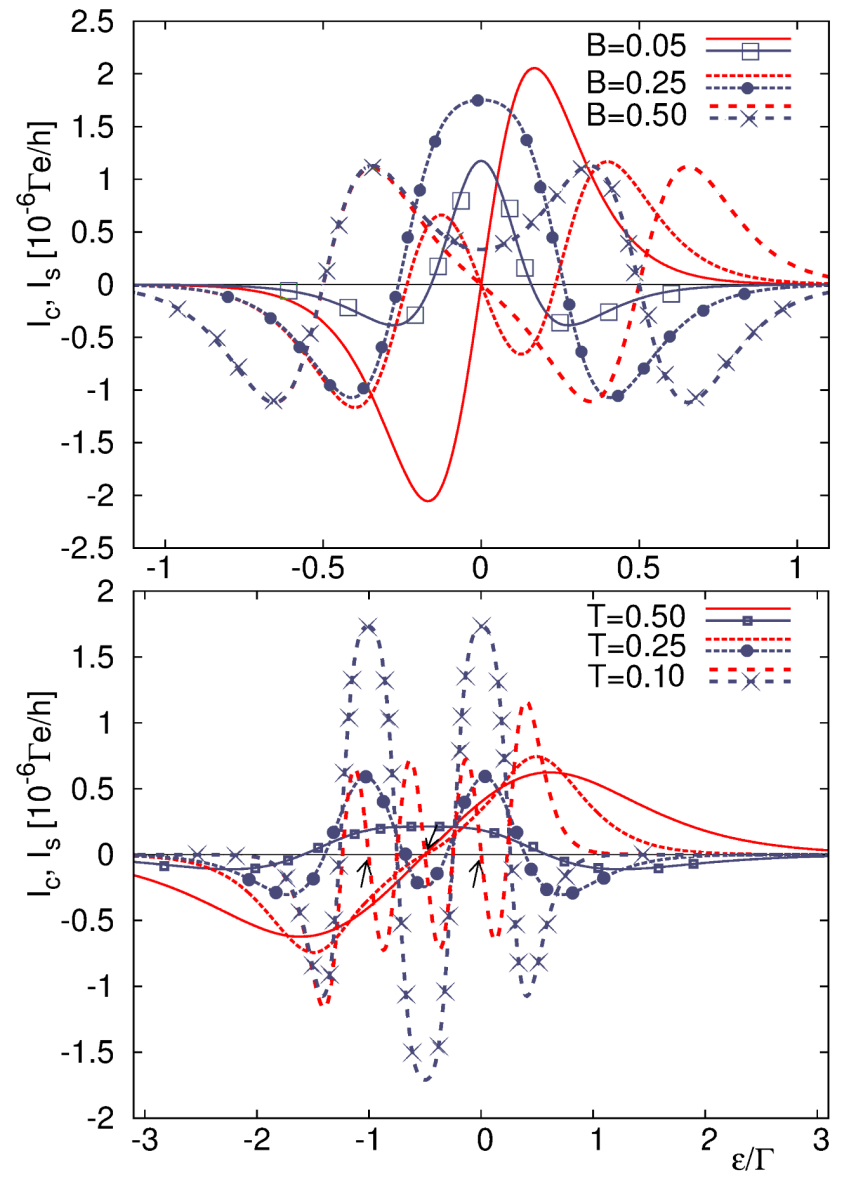

Fig. 1. Thermally induced charge (red curves) and spin (blue curves with symbols) currents vs. on dot energy level $\varepsilon_{\mathrm{d}}$ in the single quantum dot for three values of magnetic field and temperature $T=0.1 \Gamma$ (upper part). Lower part shows similar dependence in the two level quantum dot with difference between the levels $\Delta=1.0 \Gamma$ for magnetic field $B=0.25 \Gamma$ and for three different temperatures $T$ in units of $\Gamma$. 


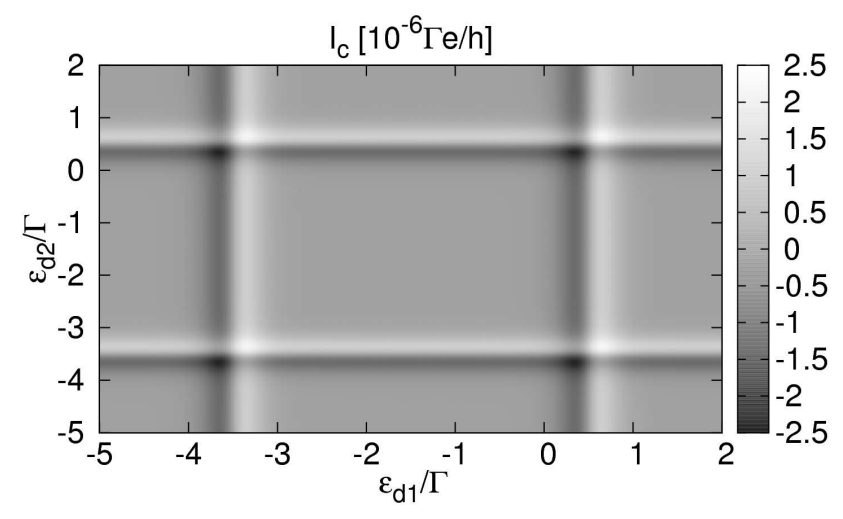

Fig. 2. Thermally induced charge current on the $\varepsilon_{d 1}-\varepsilon_{d 2}$ plane for two interacting quantum dots with $U_{1}=U_{2}=3 \Gamma$ and magnetic field $B / \Gamma=0.5$. Other parameters: $T / \Gamma=0.1, \Delta T / \Gamma=10^{-6}$, and all couplings to the electrodes equal to $\Gamma$.

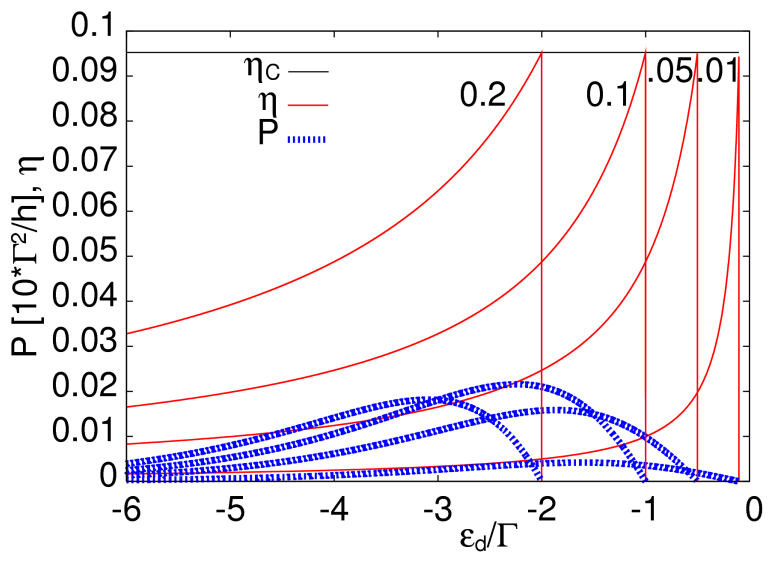

Fig. 3. (color online) The $\varepsilon_{d}$ dependence of the efficiency (red/thin solid lines) and the power (blue/dashed thick lines) of the single level quantum dot energy generator. The numbers close to thin lines denote load voltages $V$ in units of $\Gamma$. Temperatures of the electrodes $T_{\mathrm{c}}=0.95 \Gamma, T_{\mathrm{R}}=1.05 \Gamma, \eta_{\mathrm{C}}=0.095$ is the Carnot efficiency.

figure) at which charge current vanishes while spin current is maximal. Moreover, the change of the gate voltage (or $\varepsilon_{d}$ ) may reverse the direction of the spin current - the functionality which might be used in some spintronic applications. Increase of temperature may spoil the effect, additionally leading to reversal of spin current direction at the particle-hole symmetric point. The other parameters are as follows $T=0.1 \Gamma$ (upper part) and $B=0.5 \Gamma$ (lower part). We assumed symmetric couplings to the leads $\Gamma_{\mathrm{L}}=\Gamma_{\mathrm{R}}=\Gamma$. The current via double quantum dot on the $\varepsilon_{d 1}-\varepsilon_{d 2}$ plane for two interacting quantum dots is shown in Fig. 2. The figure is obtained for the parameters: $U_{1}=U_{2}=3 \Gamma$ and magnetic field $B / \Gamma=0.5, T / \Gamma=0.1$ and vanishingly small temperature difference $\Delta T / \Gamma=10^{-6}$ and with all couplings to the electrodes set equal to $\Gamma$. The analogous dependence of the spin current will be shown elsewhere [11].

\section{Double quantum dot as a thermoelectric generator}

From the practical point of view the power delivered by the engine is more important factor than the efficiency or ZT. The subject became recently a hot topic with many interesting results obtained for different nanodevices [12]. Some of the achievements have been reviewed [13]. We assume $T_{\mathrm{R}}-T_{\mathrm{L}}=\Delta T>0$ and $\mu_{\mathrm{L}}-\mu_{\mathrm{R}}=e V>0$. The efficiency of the device is defined in a standard way as a ratio of work done by the system $W=V I_{\mathrm{c}}$ in a unit time to the heat flux from the hot electrode $I_{Q}^{\mathrm{R}}$ :

$$
\eta=\frac{\dot{W}}{\dot{Q}}=\frac{V I_{\mathrm{c}}}{I_{Q}^{\mathrm{R}}} .
$$

The system may work as an energy generator or as refrigerator depending on the region in its parameters' space. Here we are mainly interested in energy harvesting. In this process the energy is taken from the environment and transformed into electric power. In Fig. 3 we present $\eta$ (thick lines) and the power $P=V I_{\mathrm{c}}$ (thin lines) as a function of $\varepsilon_{d}$ for the system with temperature $T / \Gamma=1$, temperature difference $T_{\mathrm{R}}-T_{\mathrm{L}}=0.1 \Gamma, U_{i}=0$ and the load voltages $V / \Gamma=0.01,0.05,0.1,0.25$. The vertical lines denote the limiting (maximal) value of the on-dot energy $\varepsilon_{d}$ for the device functioning as the energy generator. At that point the efficiency reaches the Carnot limit and the power vanishes as it should for reversibly operating engine. The power itself takes on the maximal value for $\varepsilon_{d}$ smaller than that corresponding to the maximal value of the efficiency.

\section{Summary and conclusions}

We have studied two-terminal device with quantum dot able to support pure spin current. The gate voltage provides a control of the direction of the spin current in the device based on two-level quantum dot in externally applied magnetic field. The device may also serve as energy generator. The efficiency of the corresponding heat to power converter depends on the parameters. It takes on maximal i.e. Carnot value for irreversible operation conditions and attains lower values at maximum power in agreement with general theory [10]. It should be stressed that contrary to the common belief $[3,6]$ the device is poor energy converter in the parameter region where its thermoelectric figure of merit is large.

\section{Acknowledgments}

This work has been partially supported by the National Science Centre under the contract DEC2011/01/B/ST3/04428. 


\section{References}

[1] A.I. Boukai, Y. Bunimovich, J. Tahir-Kheli, J.-K. Yu, W.A. Goddard III, J.R. Heath, Nature 451, 168 (2008).

[2] K. Uchida, S. Takahashi, K. Harii, J. Ieda, W. Koshibae, K. Ando, S. Maekawa, E. Saitoh, $N a-$ ture 455, 778 (2008).

[3] Y. Dubi, M. Di Ventra, Phys. Rev. B 79, 081302 (2009); P. Murphy, S. Mukerjee, J. Moore, Phys. Rev. B 78, 161406 (2008).

[4] L.I. Glazman, V. Chandrasekhar, Europhys. Lett. 19, 623 (1992).

[5] B.R. Bułka, T. Kostyrko, M. Tolea, I.V. Dinu, J. Phys. Condens. Matter 19, 255211 (2007); I. Weymann, Phys. Rev. B 78, 045310 (2008).

[6] R. Świrkowicz, M. Wierzbicki, J. Barnaś, Phys. Rev. B 80, 195409 (2009); M. Wierzbicki, R. Swirkowicz, Phys. Rev B 84, 075410 (2011); P. Trocha, J. Barnaś, Phys. Rev. B 85, 085408 (2012).
[7] M.M. Wysokinski, Acta Phys. Pol. A 122, 758 (2012); M.M. Wysokinski, J. Spalek, J. Appl. Phys. 113, 163905 (2013).

[8] T. Rejec, R. Żitko, J. Mravlje, A. Ramŝak, Phys. Rev. B 85, 085117 (2012).

[9] S. Lipiński, D. Krychowski, Phys. Rev. B 81, 115327 (2010).

[10] F.L. Curzon, B. Ahlborn, Am. J. Phys. 43, 22 (1975).

[11] B. Szukiewicz, K.I. Wysokiński, to be published.

[12] M. Esposito, K. Lindenberg, C. Van den Broeck, Euro Phys. Lett. 85, 60010 (2009).

[13] U. Seifert, Rep. Prog. Phys. 75, 126001 (2012). 